\title{
The role of adalimumab in rheumatic and autoimmune disorders: comparison with other biologic agents
}

This article was published in the following Dove Press journal:

Open Access Rheumatology: Research and Reviews

3 May 2012

Number of times this article has been viewed

\section{Andreas M Reimold \\ Dallas Veterans Affairs Medical Center and Rheumatic Diseases Division, University of Texas Southwestern Medical Center, Dallas, TX, USA}

Correspondence: Andreas M Reimold Medical Service, Dallas VA Medical Center, 4500 South Lancaster Road, Dallas, TX 75216, USA

Tel +I 2148574230

Fax +| 214857 |457

Email andreas.reimold@va.gov
Abstract: Adalimumab (ADA) is a biologic medication that dampens inflammatory pathways by binding to the cytokine tumor necrosis factor alpha. The US Food and Drug Administration has approved ADA as a medication for use in rheumatoid arthritis, psoriatic arthritis, ankylosing spondylitis, Crohn's disease, psoriasis, and juvenile idiopathic arthritis. This year marks 10 years of clinical experience with ADA. Long-term extension studies of some of the initial clinical trials, as well as data from large patient registries, are demonstrating ongoing benefit for responders. Potential side effects such as increased risk of infection, lymphoma, congestive heart failure, and demyelination continue to be examined, as the available data are not unanimous in showing an increase in incidence. In balancing both the advantages and the disadvantages of using ADA, the drug's overall effectiveness and its availability for use in patients with hepatic or renal comorbidities are weighed against the high cost. ADA is expected to have a leading role in the treatment of rheumatoid arthritis and other inflammatory conditions for years to come. Future studies will need to address the optimal sequence of disease-modifying antirheumatic drugs and biologics to use, combinations of disease-modifying antirheumatic drugs and biologics, and head-to-head comparisons of biologics in clinical trials. For those who go into clinical remission on an anti-tumor necrosis factor medication, unanswered questions remain about identifying the patients who can maintain the remission off all drugs, or at least off injected medication. Given the cost of biologic drugs, even studies that increase the interval between drug doses in well-controlled patients could provide financial benefits.

Keywords: rheumatoid arthritis, Humira ${ }^{\circledR}$, tumor necrosis factor alpha, disease-modifying antirheumatic drug

\section{Introduction}

Systemic rheumatic diseases such as rheumatoid arthritis (RA), ankylosing spondylitis (AS), psoriatic arthritis (PsA), and juvenile inflammatory arthritis have partially overlapping clinical manifestations of inflammation that are most destructive in and around joints. Given such similarities in important clinical features, the therapies used in the disorders also overlap significantly. Older therapies were generally oral disease-modifying antirheumatic drugs (DMARDs), including methotrexate (MTX), leflunomide, sulfasalazine, and hydroxychloroquine. The new medications introduced since 1999 have all been biologic drugs, meaning that they are proteins generated in vitro and purified for subcutaneous or intravenous injection. The first category of biologics has consisted of antibodies or fusion proteins that block the action of the cytokine tumor necrosis factor alpha $(\mathrm{TNF} \alpha)$ - that is, anti-TNF $\alpha$. 
By now, there are five such drugs approved in the United States: etanercept $\left(\right.$ Enbrel $\left.^{\circledR}\right)$, infliximab (Remicade $\left.{ }^{\circledR}\right)$, ADA (Humira ${ }^{\circledR}$ ), certolizumab pegol $\left(\right.$ Cimzia $\left.^{\circledR}\right)$, and golimumab (Simponi ${ }^{\circledR}$ ). Additional biologics have targeted other aspects of inflammatory pathways, including T-cell costimulation (abatacept) or T-cell costimulation and apoptosis (alefacept), B cells (rituximab), interleukin (IL)-1 (anakinra), the IL-6 receptor (tocilizumab), and IL-12 and IL-23 (ustekinumab) (Table 1). The presence of similar inflammatory pathways in Crohn's disease and plaque psoriasis has led to the inclusion of these conditions in the range of disorders treated by biologic medications.

This review will focus on ADA, a monoclonal antibody targeting TNF $\alpha$, given by subcutaneous injection. The most common interval of dosing is every 2 weeks, giving it convenience of use that has been widely accepted. ADA has been in clinical use now for a decade, allowing an assessment of its role in the therapy of multiple autoimmune diseases.

\section{Pharmacology of ADA}

ADA was discovered using phage display technology directed at a single antigen of human TNF $\alpha$. It is a fully human monoclonal antibody of the IgG1 type, containing two kappa light chains. The DNA for the antibody is expressed in the Chinese hamster ovary cell line. The antibody consists of 1330 amino acids and has a molecular weight of about $148 \mathrm{kDa} .^{1}$

The main mechanism of action for ADA is that of binding to TNF $\alpha$ and thereby blocking the interaction of TNF $\alpha$ with the p55 and p75 cell surface TNF receptors. ADA binds to both soluble and cell-bound TNF $\alpha$. In addition, in vitro experiments have demonstrated that ADA together with complement acts to lyse cells expressing TNF $\alpha$ on their surface. ADA does not bind or inactivate lymphotoxin (TNF beta).

ADA is expected to have numerous biologic effects on pathways downstream of TNF $\alpha$. One such example is downmodulation of the levels of adhesion molecules responsible for leukocyte migration (endothelial-leukocyte adhesion molecule 1, vascular cell adhesion molecule 1, and intercellular adhesion molecule 1 , with a half maximal inhibitory concentration of $1-2 \times 10-10 \mathrm{M}){ }^{1}$

ADA began clinical trials in 1997. In 2002, ADA received its first US Food and Drug Administration (FDA) approval for RA patients with prior DMARD failure. The drug is marketed as $40 \mathrm{mg}$ of product in single-use containers consisting of either a prefilled syringe or a pen. The needle cover of the prefilled syringe contains latex rubber, which is a consideration for those allergic to latex. The drug is considered stable for up to 24 months at $2^{\circ} \mathrm{C}-8^{\circ} \mathrm{C}$. Pharmacokinetic studies have revealed a half-life of about 2 weeks (range 10-20 days) after a $40 \mathrm{mg}$ dose.

Even though ADA is a fully human monoclonal antibody, antibodies against ADA occur in clinical practice. In a recent study, $76(28 \%)$ of 278 RA patients receiving ADA for 3 years were positive for anti-ADA antibodies, and $67 \%$ of the positives developed antibodies within 28 weeks of starting therapy. ${ }^{2}$ Patients with anti-ADA antibodies achieved remission less often (28-joint disease activity score [DAS28] < 2.6) than those without (hazard ratio [HR], 7.1; 95\% confidence interval [CI], 2.1-23.4; $P<0.001)$. Clearly, many instances of anti-ADA antibody formation do not have a detrimental impact on therapeutic

Table I Indications of biologic medications approved by the US Food and Drug Administration

\begin{tabular}{|c|c|c|c|c|c|c|c|c|}
\hline Generic & Trade & Target & RA & PsA & AS & CD & Ps & JIA \\
\hline \multicolumn{9}{|l|}{ TNF $\alpha$ antagonists } \\
\hline Adalimumab & Humira $^{\circledR}$ & TNF $\alpha$ & $\checkmark$ & $\checkmark$ & $\checkmark$ & $\checkmark$ & $\checkmark$ & $\checkmark$ \\
\hline Infliximab & Remicade $^{\circledR}$ & $\mathrm{TNF} \alpha$ & $\checkmark$ & $\checkmark$ & $\checkmark$ & $\checkmark$ & $\checkmark$ & \\
\hline Etanercept & Enbrel $^{\circledR}$ & $\mathrm{TNF} \alpha$ & $\checkmark$ & $\checkmark$ & $\checkmark$ & & $\checkmark$ & $\checkmark$ \\
\hline Certolizumab pegol & $\mathrm{Cimzia}^{\circledR}$ & $\mathrm{TNF} \alpha$ & $\checkmark$ & & & $\checkmark$ & & \\
\hline Golimumab & Simponi ${ }^{\circledR}$ & TNF $\alpha$ & $\checkmark$ & $\checkmark$ & $\checkmark$ & & & \\
\hline \multicolumn{9}{|l|}{ Other biologics } \\
\hline Abatacept & Orencia ${ }^{\circledR}$ & T-cell costimulation & $\checkmark$ & & & & & $\checkmark$ \\
\hline Alefacept & Amevive ${ }^{\circledR}$ & $\mathrm{T}$ cell & & & & & $\checkmark$ & \\
\hline Anakinra & Kineret $^{\circledR}$ & IL-I & $\checkmark$ & & & & & \\
\hline Tocilizumab & Actemra $^{\circledR}$ & IL-6 receptor & $\checkmark$ & & & & & $\checkmark$ \\
\hline Ustekinumab & Stelara ${ }^{\circledR}$ & IL-I2/23 & & & & & $\checkmark$ & \\
\hline Rituximab & Rituxan $^{\circledR}$ & B cell & $\checkmark$ & & & & & \\
\hline
\end{tabular}

Abbreviations: AS, ankylosing spondylitis; CD, Crohn's disease; IL, interleukin; JIA, juvenile idiopathic arthritis; Ps, psoriasis; PsA, Psoriatic arthritis; RA, rheumatoid arthritis; TNF $\alpha$, tumor necrosis factor alpha. 
response, but it represents one possible explanation when effectiveness is lost.

\section{Efficacy studies \\ RA}

The FDA first approved ADA in 2002 for treatment of moderate to severe RA in patients with an inadequate response to DMARD therapy; ADA was approved in 2005 for first-line use in RA. Five randomized, double-blind clinical trials were presented between 2003 and 2006 to assess the efficacy of ADA in combination with MTX, as monotherapy, or with standard oral antirheumatic therapy. The first of these, the ARMADA (Anti-TNF Research Study Program of the Monoclonal Antibody D2E7 in Patients with Rheumatoid Arthritis) trial, will be discussed here, while further trials of efficacy in long-duration RA (DEO11 and DEO19), safety (STAR [Safety Trial of Adalimumab in Rheumatoid Arthritis]), and early RA in MTX-naïve patients (PREMIER) are summarized in Table 2.

The ARMADA trial was a 24-week randomized, double-blind, placebo (PBO)-controlled trial to study the combination of ADA plus MTX in RA patients with longstanding disease (mean duration 12.3 years) who had an inadequate response to oral DMARD therapy (MTX and up to three other drugs). ${ }^{3}$ The study population consisted of 271 patients, $76.8 \%$ of whom were female, with a mean age of 55.5 years. Disease activity was high, with a mean tender joint count of 28.9 and a mean swollen joint count of 17.2. The four treatment arms comprised roughly equal numbers receiving MTX with PBO, MTX with ADA $20 \mathrm{mg}$ every other week (eow), MTX with ADA $40 \mathrm{mg}$ eow, or MTX with ADA $80 \mathrm{mg}$ eow. The primary efficacy endpoint was response to the American College of Rheumatology $20 \%$ improvement criteria (ACR20) at 24 weeks, with the ACR 50 and ACR70 (ACR 50\% and 70\% improvement criteria, respectively) response rates as secondary endpoints. An ACR20 response is defined as at least 20\% improvement in both tender and swollen joint counts, and in at least three of five core set measurements: (1) physical function, (2) patient assessment of pain, (3) patient global assessment of disease activity, (4) physician global assessment (PGA), and (5) acute phase reactant (erythrocyte sedimentation rate or C-reactive protein). The analysis was by intention to treat and included all patients who received at least one dose of ADA or PBO.

The main result of the ARMADA trial demonstrated statistically significant responses for ADA arms at 24 weeks when compared with the PBO arm: ACR20 response achieved in 65\% (ADA $40 \mathrm{mg}$ eow plus MTX) versus 13\% (PBO plus MTX); ACR50 response achieved in 52\% versus 7\%; and ACR 70 response achieved in $24 \%$ versus $3 \%$. There was a dose response in ACR scores going from 20 to $40 \mathrm{mg}$ of ADA, with no further improvement using the $80 \mathrm{mg}$ dose. Similar numbers of ADA- and PBO-treated patients reported adverse events.

Overall, studies on ADA have demonstrated effectiveness in RA patients with early disease not previously treated with MTX, and also in long-standing disease that has failed to respond to oral DMARDs. Study patients receiving ADA had inhibition of radiographic progression, they had improvement and stabilization of their Health Assessment Questionnaire scores, and they tolerated the treatment as well as comparators not on ADA.

\section{PsA}

The FDA approved ADA in 2005 for the treatment of PsA, reducing signs and symptoms, inhibiting progression of structural damage, and improving physical function. The results of two randomized, double-blind, PBO-controlled studies in patients with moderate to severe PsA have been published.

The ADEPT (Adalimumab Effectiveness in Psoriatic Arthritis Trial) study included 313 adult PsA patients representing all five subtypes of PsA distribution but predominantly polyarticular (RA-like) involvement in 210 patients. ${ }^{8}$ The disease was active (over three swollen and tender joints) and there had been an inadequate response to nonsteroidal anti-inflammatory drug (NSAID) therapy. About half the patients were receiving stable MTX therapy. Treatment was a 24-week course with ADA $40 \mathrm{mg}$ eow or PBO. The primary endpoints were ACR20 responses at week 12 and radiographic change by modified total Sharp score (mTSS) for ADA at week 48 compared with PBO at week 24. An openlabel extension was begun after week 24 , in which all patients received ADA. The results showed that an ACR20 response was achieved at week 24 by $57 \%$ of ADA-treated patients and $15 \%$ of PBO-treated patients $(P<0.001)$. Radiographic progression was assessed at the hands, wrists, and feet. ADAtreated patients showed a decrease of 0.2 in mTSS at week 48 , while PBO-treated patients had an increase of 0.9 in mTSS at week $24(P<0.001)$. The Health Assessment Questionnaire Disability Index score had a mean decrease from baseline of 0.4 points $(49 \%)$ in the ADA group, while it decreased 0.1 point $(3 \%)$ in the $\mathrm{PBO}$ group $(P<0.001)$.

A further clinical trial was a 12-week, PBO-controlled, double-blind, randomized study of 100 PsA patients with inadequate response to NSAIDs or oral DMARDs. 


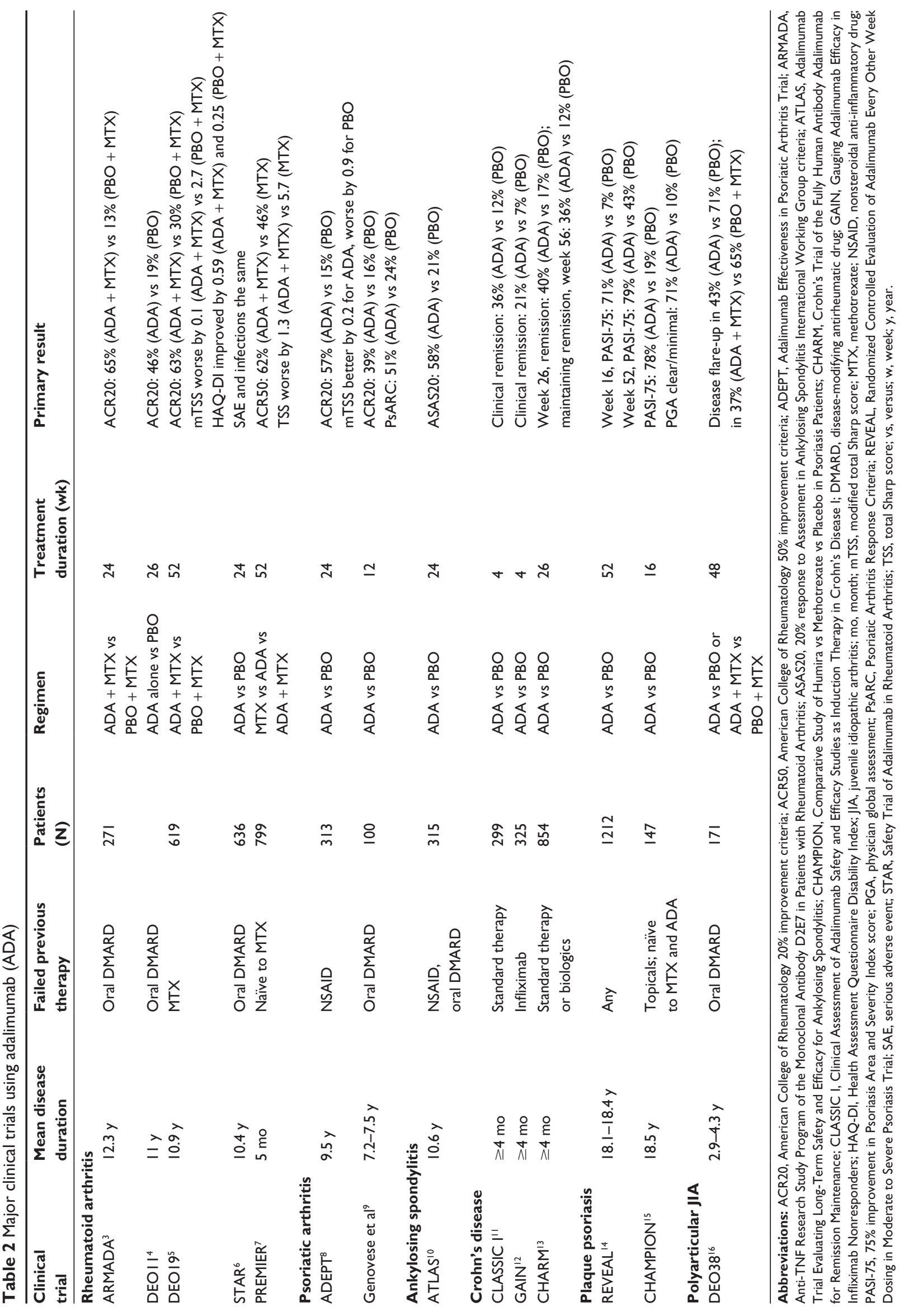


Treatment was with ADA $40 \mathrm{mg}$ eow versus PBO, followed by open-label ADA for all participants for a further 12 weeks. $^{9}$ At week 12, 39\% of the ADA-treated patients had an ACR20 response compared with $16 \%$ of PBO-treated patients $(P=0.012)$, and the Psoriatic Arthritis Response Criteria response was $51 \%$ for ADA-treated patients and $24 \%$ for PBO-treated patients $(P=0.007)$. After both groups were given open-label ADA for weeks 13-24, ACR20 responses were found in $65 \%$ of those originally receiving ADA, and $57 \%$ of those originally receiving $\mathrm{PBO}$. Therefore, there was substantial further improvement in ACR responses between weeks 13 and 24 of therapy.

\section{AS}

The FDA approved ADA for the treatment of AS in 2006, and its pivotal trial was the ATLAS (Adalimumab Trial Evaluating Long-Term Safety and Efficacy for Ankylosing Spondylitis) study. ${ }^{10}$ ATLAS was a 24-week, randomized, double-blind, PBO-controlled trial of 315 AS patients with active disease despite traditional therapy with glucocorticoids, NSAIDs, analgesics, MTX, or sulfasalazine. Treatment in the study compared ADA $40 \mathrm{mg}$ eow with PBO. In contrast to RA, different instruments exist for the evaluation of AS disease activity and response to therapy. In ATLAS, active disease was defined as meeting two of three criteria: a Bath Ankylosing Spondylitis Disease Activity Index score $\geq 4$, total back pain score $\geq 4 \mathrm{~cm}$ (on a $0-10 \mathrm{~cm}$ visual analog scale [VAS]), and morning stiffness lasting 1 hour or more. The primary efficacy endpoint in the study was the percentage of patients achieving an ASAS20 response. ASAS20 indicates a 20\% response to the Assessment in Ankylosing Spondylitis International Working Group criteria, which consist of (1) patient's global assessment of disease activity (by VAS); (2) total back pain (by VAS); (3) inflammation (morning stiffness and duration by VAS); and (4) Bath Ankylosing Spondylitis Functional Index, representing an average score from 10 questions on functional disability by week 12 .

The results of the ATLAS trial showed that ADA-treated patients met the primary endpoint at 12 weeks significantly more frequently than PBO-treated patients: 58\% versus $21 \%$, respectively $(P<0.001)$. There was a very rapid onset of action, with the benefits of ADA use seen by week 2 of the trial (ADA response 42\%; PBO response $16 \% ; P<0.001)$. Even patients with total spinal ankylosis achieved ASAS20 responses at week 12 (in three of six cases, compared with zero of five cases for PBO), although the low patient numbers did not reach statistical significance.
Efficacy was also demonstrated for reduction in enthesitis among ADA-treated patients (Maastricht ankylosing spondylitis enthesitis score decreased by 2.7 for those receiving $\mathrm{ADA}$ and by 1.3 for those receiving $\mathrm{PBO}$ $[P=0.02])$. All responses were maintained throughout the 24-week trial.

\section{Crohn's disease}

The FDA approved ADA in 2007 to treat moderate to severe Crohn's disease in those with an inadequate response to conventional therapy and in those who have lost response to or are intolerant of infliximab. Three major clinical trials have been conducted to study ADA in patients with moderate to severe active Crohn's disease: CLASSIC I (Clinical Assessment of Adalimumab Safety and Efficacy Studies as Induction Therapy in Crohn's Disease I), GAIN (Gauging Adalimumab Efficacy in Infliximab Nonresponders), and CHARM (Crohn's Trial of the Fully Human Antibody Adalimumab for Remission Maintenance) (summarized in Table 2). All were double-blind, randomized, PBO-controlled trials. ${ }^{11-13}$ Two of the trials studied the induction of clinical remission after 4 weeks of therapy, while the third focused on maintaining remission in a 56-week trial. For these studies, each case was scored with the Crohn's Disease Activity Index (CDAI), a weighted composite score of eight clinical factors: (1) daily number of liquid or very soft stools, (2) severity of abdominal pain, (3) level of general well-being, (4) number of complications, (5) use of antidiarrheal agents, (6) presence of abdominal mass, (7) hematocrit, and (8) decrease in body weight. A clinical remission was defined as a CDAI score below 150 points.

In the CLASSIC I study, 299 anti-TNF-naïve patients with moderate to severe active Crohn's disease (mean CDAI score 298) of at least 4 months' duration were randomized to receive one of the following treatments at weeks 0 and 2: (1) PBO both times, (2) ADA $160 \mathrm{mg}$ at week 0 and $80 \mathrm{mg}$ at week 2, (3) ADA $80 \mathrm{mg}$ at week 0 and $40 \mathrm{mg}$ at week 2, or (4) ADA $40 \mathrm{mg}$ at week 0 and $20 \mathrm{mg}$ at week 2. ${ }^{11}$ The primary endpoint was induction of clinical remission (CDAI score $<150$ ) at week 4. Background therapies were permitted, including 5-aminosalicylates, corticosteroids, immunomodulators, and antibiotics. The results demonstrated clinical remission in $36 \%$ of the ADA $160 / 80 \mathrm{mg}$ group compared with $12 \%$ of the PBO group $(P<0.001)$. There was a dose response with the other two ADA groups showing clinical remission in 24\% (ADA 80/40 mg) and $18 \%$ (ADA 40/20 mg), but these were not significant when compared with $\mathrm{PBO}$. 
The CHARM study was designed to evaluate the maintenance of clinical remission. ${ }^{13}$ The 56-week trial of 854 patients with moderate to severe active Crohn's disease began with an open-label period of ADA $80 \mathrm{mg}$ (week 0) and $40 \mathrm{mg}$ (week 2). By week 4, there were 499 (58\%) who had achieved a CDAI score reduction of $\geq 70$ points. These 499 patients were randomized to PBO, ADA $40 \mathrm{mg}$ weekly, or ADA $40 \mathrm{mg}$ eow. The primary endpoints were maintenance of clinical remission (CDAI score $<150$ ) at weeks 26 and 56. The results showed that at week $26,40 \%$ of patients receiving $\mathrm{ADA}$ eow and $17 \%$ of patients receiving $\mathrm{PBO}$ achieved clinical remission $(P<0.001)$, while at week 56, remission was seen in $36 \%$ of patients receiving ADA and $12 \%$ of patients receiving PBO $(P<0.001)$. There was no additional advantage to using ADA every week. Also, those not responding by week 12 did not have significantly more responses with a longer duration of therapy.

\section{Chronic plaque psoriasis}

In 2008, ADA received FDA approval for the treatment of moderate to severe chronic plaque psoriasis in those patients who are candidates for systemic therapy or phototherapy, and when other systemic therapies are medically less appropriate. Two randomized, double-blind, PBO-controlled studies are the pivotal trials for ADA use in chronic plaque psoriasis (summarized in Table 2). ${ }^{14,15}$

REVEAL (Randomized Controlled Evaluation of Adalimumab Every Other Week Dosing in Moderate to Severe Psoriasis Trial) was a 52-week trial that involved 1212 patients with $\geq 10 \%$ body surface area involvement, a PGA of at least moderate disease severity, and a Psoriasis Area and Severity Index (PASI) score $\geq 12 .{ }^{14}$ The study was designed to include three treatment periods: (1) patients were randomized to receive 16 weeks of either ADA $80 \mathrm{mg}$ once and then $40 \mathrm{mg}$ eow or PBO; (2) patients who had achieved a PASI-75 response (75\% improvement in PASI score) in the first period next received open-label ADA at $40 \mathrm{mg}$ eow for 17 weeks; (3) patients who maintained a PASI-75 response at week 33 and had received ADA in the first period were re-randomized to 19 weeks of ADA $40 \mathrm{mg}$ eow, or PBO. The primary endpoints at the end of the first period (week 16) were the proportion of patients achieving a PASI-75 response and the proportion of patients achieving clear or minimal skin disease on the six-point PGA scale. The results demonstrated that $71 \%$ of ADA-treated patients achieved the PASI-75 response at week 16, compared with $7 \%$ of PBO-treated patients $(P<0.001)$. In addition, $62 \%$ of ADA-treated patients achieved a PGA score of clear or minimal at week 16 , compared with $4 \%$ of patients in the PBO group $(P<0.001)$. At 52 weeks, patients continuing to receive ADA had superior maintenance of efficacy to those re-randomized to PBO: $5 \%$ of those receiving ADA lost PASI-75 response compared with $28 \%$ of those receiving PBO $(P<0.001)$.

The CHAMPION (Comparative Study of Humira versus Methotrexate versus Placebo in Psoriasis Patients) study was a phase III, 16-week trial to evaluate 147 patients with chronic plaque psoriasis randomized to receive $\mathrm{ADA}$ or $\mathrm{PBO}$. The ADA patients received ADA $80 \mathrm{mg}$ at week 0, followed by $40 \mathrm{mg}$ eow. ${ }^{15}$ Primary endpoints were the same as in the REVEAL study: the proportion of patients achieving a PASI-75 response relative to baseline, and clear or minimal disease on the six-point PGA scale. The results showed that $78 \%$ of ADA-treated patients achieved a PASI-75 response, compared with $19 \%$ of PBO-treated patients $(P<0.001)$, and $71 \%$ of ADA-treated patients achieved a PGA of clear or minimal compared with $10 \%$ of PBO-treated patients $(P<0.001)$. These results confirmed the findings of ADA's efficacy at 16 weeks in chronic plaque psoriasis patients.

\section{Polyarticular juvenile idiopathic arthritis}

The first pediatric indication for ADA was approved in 2008 , in the treatment of moderate to severe polyarticular juvenile idiopathic arthritis (JIA), alone or in combination with MTX, for patients aged 4 years and older. The pivotal trial was a multicenter, randomized, double-blind study in 171 children with polyarticular JIA. ${ }^{16}$ Patients were divided into two groups, MTX-treated or non-MTX-treated and all had moderate to severe disease despite previous treatment with NSAIDs, analgesics, corticosteroids, or oral DMARDs. The trial lasted 48 weeks, beginning with a 16-week openlabel phase in which all patients received ADA at $24 \mathrm{mg} / \mathrm{m}^{2}$ (maximum of $40 \mathrm{mg}$ ) eow. This was followed by a 32-week double-blind period in which those who had achieved a Pediatric (Pedi) ACR30 response in the open-label phase were randomized to continue ADA or switch to PBO, until completion of the trial or until a disease flare-up occurred. The results after the 16-week open-label phase showed $94 \%$ of patients receiving MTX plus ADA achieved the Pedi ACR30 response, while only $74 \%$ of those receiving ADA without MTX did so. In the double-blind phase, significantly fewer patients taking ADA experienced a disease flare-up, both with MTX ( $37 \%$ of those also receiving ADA compared with $65 \%$ of those receiving PBO, $P=0.015$ ) and without MTX (43\% of those receiving ADA compared with $71 \%$ of those receiving PBO, $P=0.031$ ). The Pedi ACR30, Pedi ACR50, 
and Pedi ACR70 responses were reached by a significantly greater proportion in the MTX plus ADA group at week 48 than the MTX plus PBO group (eg, Pedi ACR70 response in $63 \%$ of those in the MTX plus ADA group compared with $27 \%$ of those in the MTX plus PBO group, $P=0.002$ ), and the trend was similar for the non-MTX groups, although it did not reach statistical significance. The small size of the groups available for analysis (between 28 and 38 individuals) and rigorous classification of nonresponders made it difficult to show statistical significance for some comparisons. Adverse reactions were generally similar to those seen in adults, but some adverse reactions seen less commonly in adults were highlighted: neutropenia, streptococcal pharyngitis, elevated transaminases, myositis, metrorrhagia, and appendicitis.

\section{Long-term use}

Many clinical trials using ADA were designed to have a relatively short duration of less than 1 year. Additional experience about effectiveness and safety has been described for some indications using long-term open-label follow-up studies, and, increasingly, from clinical experience compiled in large patient registries. Such studies are not rigorously controlled, but they do provide additional information of medication use in real-world situations.

\section{RA}

Several clinical trials of ADA have had long-term follow-up in open-label extensions. While such studies select for patients who continue to respond to ADA, they do give information on long-term efficacy and tolerability.

The ARMADA trial of ADA plus MTX in RA was a 24-week study with open-label follow-up reported in 168 patients (64\% of those who received ADA in the study). ${ }^{17}$ The observation period was up to 4 years. Efficacy achieved at 24 weeks in the study was maintained. Clinical remission (DAS28 < 2.6) was reached by $43 \%$ of patients. Reduction of concomitant corticosteroid or MTX therapy did not affect long-term efficacy. In addition, adverse events occurred at similar rates in the initial blinded period and during the open-label extension.

Recently, 10-year results have been presented for the openlabel extension after the DEO19 trial, a phase III, randomized, PBO-controlled trial in active, advanced RA with an inadequate response to MTX. ${ }^{18}$ Patients had been randomized to MTX plus 1 year of ADA $40 \mathrm{mg}$ eow, $20 \mathrm{mg}$ weekly, or PBO injections. The clinical and radiographic superiority of ADA plus MTX over PBO plus MTX were demonstrated, and all trial completers could enter the open-label extension of ADA
$40 \mathrm{mg}$ eow plus MTX for an additional 9 years. The results showed that $202(32.6 \%)$ of the original 619 patients continued with treatment through year 10. Outcomes on the DAS28 and on the Health Assessment Questionnaire Disability Index showed no significant difference at year 10, regardless of the initial randomization group. However, the radiographic damage as measured by change in mTSS was lower in the initial $40 \mathrm{mg}$ eow ADA group compared with the initial PBO group ( 0.7 versus 6.2 , respectively, $P=0.005)$. This was a reflection of the damage that occurred in the first year of the trial, with the rate of radiographic progression being similar in all groups once they took ADA starting in year 2 .

Long-term results are also available for patients with early RA in the PREMIER study. ${ }^{19}$ Through 8 years, 299 (37.4\%) of the original 799 patients remained under observation in an open-label extension study. About $60 \%$ of patients completing 8 years of therapy achieved DAS28 remission. Patients initially randomized to ADA plus MTX had less radiographic progression than those randomized to either ADA or MTX monotherapy, as measured by lower mean change in mTSS $(P<0.001)$. Again, the lower level of structural damage accrued during the initial 2-year blinded period by ADA plus MTX patients accounted for these differences, with rates of further radiographic progression similar in all groups once open-label ADA plus MTX was started.

\section{PsA}

An open-label extension in 285 patients who completed the double-blind, PBO-controlled portion of the ADEPT study was analyzed after 2 years of treatment. ${ }^{20}$ Patients maintained the improvement in the inhibition of radiographic progression, joint inflammation, and skin disease compared with the 24-week time point. In a study from the British Society for Rheumatology Biologics Register, an estimated 1-year drug survival on anti-TNF medication (infliximab, $\mathrm{n}=162$; etanercept, $n=316$; or ADA, $n=88$ ) was reported as $82 \%$, while even those switching to a second anti-TNF agent had $72 \%$ 1-year drug survival on the second drug. ${ }^{21}$ Anti-TNF discontinuation was more common in infliximab than etanercept recipients (HR, 2.8; 95\% CI, 2.1-3.7), and etanercept and ADA had comparable discontinuation rates.

\section{AS}

Patients in the ATLAS trial have now been followed for up to 5 years in an open-label extension. ${ }^{22}$ Of the 311 patients who received at least one dose of ADA, 202 (65\%) completed 5 years of the study. There were 125 patients who had been originally randomized to receive ADA and who remained 
on the drug for 5 years. Of these patients, $70.4 \%$ maintained an ASAS40 response, 50.8\% maintained an ASAS partial remission, and $60.5 \%$ maintained inactive disease (as defined by the Ankylosing Spondylitis Disease Activity Score) after 5 years. The strongest predictor of remission at years 1 and 5 was achieving remission at 12 weeks of treatment. Other factors such as age, gender, and human leukocyte antigen B27 status were not associated. Adverse event rates were comparable with those in previous ADA trials.

A separate report studying ATLAS patients along with a Canadian AS study after 2 years of treatment made the important observation that treatment with ADA did not slow radiographic progression. ${ }^{23}$ Patients who had received ADA were compared with a historic anti-TNF-naïve cohort (Outcome in Ankylosing Spondylitis International Study cohort) using the modified Stoke Ankylosing Spondylitis Spinal Score method. Mean changes in the modified Stoke Ankylosing Spondylitis Spinal Score from baseline to 2 years were 0.9 for the Outcome in Ankylosing Spondylitis International Study cohort and 0.8 for ADA-treated patients $(P=0.771)$. Nevertheless, an analysis of ATLAS patients with total spinal ankylosis demonstrated ASAS20 responses in three of six patients receiving ADA and zero of six receiving PBO. ${ }^{24}$ After switching to open-label ADA, eight of eleven ankylosed patients achieved an ASAS20 response with 1 year of ADA treatment, demonstrating benefit for the signs and symptoms of active disease.

\section{Crohn's disease}

The 1-year CHARM trial had a 2-year open-label extension phase, with a recent analysis examining 206 patients randomized to $\mathrm{ADA}$ who also took corticosteroids at baseline. ${ }^{25}$ Rates of steroid-free remission were sustained at 3 years in those who achieved them at 1 year: steroid-free remission, 26\% (1 year) and 23\% (3 years); steroid-sparing ( $\geq 50 \%$ steroid dose reduction) remission, 29\% (1 year) and $25 \%$ (3 years); and steroid-free response, 32\% (1 year) and $28 \%$ ( 3 years). These results were described as modest but clinically meaningful. A separate single-center experience in a historical cohort study found an annual risk of loss of response to ADA in Crohn's disease patients of $18 \%$, with risk factors of extraintestinal manifestations and previous exposure to a different anti-TNF agent. ${ }^{26}$

\section{Psoriasis}

The initial clinical trials on ADA use in the treatment of plaque psoriasis were generally of 6 months' duration or less. The major long-term study in PsA, ADEPT, is therefore of interest for including some measures of skin disease. ${ }^{8}$ Of the original 298 patients who completed the 24-week doubleblind portion of the trial, 285 went on to an open-label extension, receiving ADA $40 \mathrm{mg}$ eow for up to 120 weeks. At week 48, patients who received ADA throughout the trial had response rates for PASI-50 of 67\%, PASI-75 of $58 \%$, PASI-90 of $46 \%$, and PASI- 100 of $33 \%$. In those who continued ADA, the percentage of patients achieving these PASI scores changed by $\leq 10 \%$ between weeks 48 and 104.

Of the patients who had continuously taken ADA during the 12 months of the REVEAL phase III clinical trial, 233 entered an open-label extension and could be evaluated after a total of 18 months of ADA therapy. ${ }^{14}$ Of these patients, $85 \%, 59 \%$, and $35 \%$ achieved PASI-75, PASI-90, and PASI-100 response rates, respectively, at month 12 , and these response rates were maintained at month 18 , achieved by $87 \%, 63 \%$, and $34 \%$ of patients, respectively. Data from the ADEPT study also allow an estimate of longer-term drug persistence and efficacy for ADA. It was found that $5 \%$ of study patients treated continuously with ADA over a year had a loss of response. On the other hand, $72 \%$ of patients who stopped ADA as part of the trial protocol had sustained efficacy 19 weeks later.

\section{Comparison of ADA with other biologics}

Randomized controlled trials of anti-TNF agents leave little doubt of their general effectiveness in the conditions for which they have received FDA approval. However, comparisons of biologic medications rely almost exclusively on indirect comparisons, as the only head-to-head trial available studied infliximab compared with abatacept. ${ }^{27}$ In considering the properties specifically of ADA compared with other treatments, two areas will be reviewed: effectiveness and side effects.

\section{RA}

Evidence comparing the effectiveness of ADA compared with other biologic medications in RA is exclusively indirect. One broad indication of a drug's effectiveness and tolerability is the persistence on medication. In $2418 \mathrm{RA}$ patients from the Rheumatoid Arthritis Disease-Modifying Antirheumatic Drug Intervention and Utilization Study registry, persistence on three of the anti-TNF medications during a first course of use was found to be similar: $48 \%$ of those receiving ADA, $48 \%$ of those receiving infliximab, $51 \%$ of those receiving etanercept. ${ }^{28}$ The Dutch Rheumatoid Arthritis 
Monitoring registry studied RA patients beginning an antiTNF drug for the first time..$^{29}$ Over 12 months, ADA-treated and etanercept-treated patients had a statistically significant greater improvement in DAS28 and SF-36 ${ }^{\circledR}$ (a multi-purpose, short-form health survey) physical component score than infliximab. The ADA group also had a larger improvement in DAS28 than the etanercept group $(P=0.031)$. In early RA, a meta-analysis found consistent benefit of using a biologic agent with MTX, rather than MTX alone, in terms of achieving clinical remission (relative risk [RR], 1.74; 95\% CI, 1.54-1.98) and X-ray nonprogression (RR, 1.30; 95\% CI, 1.01-1.63). ${ }^{30}$ However, there was no benefit of infliximab, ADA, etanercept, or abatacept over the others. Mixed treatment comparisons of short-term (6-month) efficacy in RA have shown an advantage for certolizumab pegol in achieving an ACR20, ACR50, or ACR70 response, as compared with most biologic DMARDs, including ADA, and an advantage for etanercept over ADA in achieving ACR20 and ACR50 responses. ${ }^{31} \mathrm{~A}$ meta-analysis of 13 trials in RA assessed responses at 6 months and found no difference in efficacy among infliximab, ADA, or etanercept in achieving ACR20 or ACR50 responses. ${ }^{32} \mathrm{~A}$ further meta-analysis included 21 randomized, $\mathrm{PBO}$-controlled trials, eight using ADA, seven with infliximab, and six with etanercept. ${ }^{33}$ In short-term treatment (12-30 weeks) etanercept showed the highest likelihood of achieving an ACR20 or ACR50 response, while ADA had the highest risk ratio for an ACR70 response (risk ratio, 5.28; 95\% CI, 3.76-7.64). For longterm treatment (1-3 years), ADA had the highest risk ratio for all levels of ACR response: 1.85 (95\% CI, 1.07-3.19) for ACR20, 2.80 (95\% CI, 1.16-6.77) for ACR50, and 3.23 (95\% CI, 1.37-7.61) for ACR70.

There are numerous limitations to the available clinical trials and indirect comparisons among biologic agents. In studies of the Dutch Rheumatoid Arthritis Monitoring registry of RA patients, the effectiveness of anti-TNF agents exceeded the effectiveness found in clinical practice, with the observation that patients with a lower disease activity and different (often more severe) comorbidity profile were routinely begun on biologic therapies in the clinics but would not qualify for most clinical trials. ${ }^{34}$ Meta-analyses and mixed treatment comparisons restrict which studies to include and therefore analyze an even smaller subset of all candidates for biologic therapy. Variations in study design including patient characteristics, treatment regimens, and differing outcome measures mean individual clinical trials are not directly comparable. Ultimately, direct comparative randomized clinical trials of biologics, compared against other biologics and against oral DMARDs, are still needed. Studies of sequential use of biologics in patients who have failed a previous biologic medication are also lacking.

\section{PsA}

A random effects meta-analysis of six randomized controlled trials was performed in PsA, including 982 patients receiving ADA, infliximab, or etanercept. ${ }^{35}$ The results showed similar effectiveness among all three drugs for Psoriatic Arthritis Response Criteria and ACR20, ACR50, and ACR70 responses. There were also similar rates of withdrawal for all three. Another study of the same three anti-TNF medications made a mixed treatment comparison using ACR20 responses in four pivotal trials for these medications in PsA. ${ }^{36}$ Here, all three drugs were superior to $\mathrm{PBO}$ in patients unresponsive to nonbiologic treatments, and etanercept provided the greatest likelihood of an ACR20 response compared with PBO, although the direct comparison with ADA and infliximab was not statistically significant.

\section{AS}

Recent studies have investigated drug survival of three antiTNF medications (ADA, infliximab, and etanercept) in AS. In a study of 243 Swedish TNF inhibitor-naïve patients, the 2 -year drug continuation rate was found to be $74 \% .{ }^{37}$ The presence of peripheral arthritis and male sex predicted continuation of the anti-TNF medication, but no advantage was indicated for any of the three anti-TNF drugs over the others. In the Danish nationwide rheumatologic database DANBIO, 842 anti-TNF $\alpha$-naïve patients had a median drug survival of 4.3 years ( $53 \%$ received infliximab, $29 \%$ received ADA, and 18\% received etanercept). ${ }^{38}$ Baseline characteristics associated with longer drug survival were male sex; a high C-reactive protein level, $>14 \mathrm{mg} / \mathrm{L}$; and low VAS score for fatigue. The age and use of a specific anti-TNF medication or of MTX were not significant.

Formation of antibody to anti-TNF $\alpha$ medication was studied in 60 AS patients. ${ }^{39}$ Antibodies were detected in $20 \%$ of 20 patients receiving infliximab, $30 \%$ of 20 patients receiving $\mathrm{ADA}$, and $0 \%$ of 20 patients receiving etanercept. Antibody formation was related to decreased effectiveness and early discontinuation of anti-TNF treatment in this small study. Other studies have looked at responses after switching anti-TNF medication. In a large retrospective study of 1619 AS patients taking anti-TNF medication, 38 (2.34\%) switched to ADA after failing etanercept or infliximab. ${ }^{40}$ Regardless of the reason for switching to ADA, the survival curves for ADA as a second anti-TNF agent were significantly 
better than the survival curves for these patients on their first anti-TNF drug $(P<0.0001)$. This supports the common clinical practice of switching to a second anti-TNF medication if the first one has lost effectiveness or has caused side effects.

\section{Psoriasis}

A systematic review and meta-analysis through January 2008 compared 16 double-blind PBO-controlled trials in the treatment of moderate to severe skin psoriasis. ${ }^{41}$ The highest efficacy was presented as a risk difference of pooled PASI-75 response rates, seen in 76\% of patients treated with infliximab, $59 \%$ of patients treated with ADA, $44 \%$ of those treated with high-dose (50 mg twice weekly) etanercept, and $30 \%$ of those treated with etanercept $50 \mathrm{mg}$ weekly. A further network meta-analysis of randomized controlled trials determined the probability of response and RR of therapy compared with $\mathrm{PBO} .{ }^{42}$ Here, the predicted mean probability of PASI-50 response was $93 \%$ for infliximab, $90 \%$ for ustekinumab, $81 \%$ for ADA, 76\% for etanercept $50 \mathrm{mg}$, and $13 \%$ for PBO. Therefore, all these biologics were far superior to $\mathrm{PBO}$.

In an effort to compare effectiveness without a headto-head trial, a new method of adjusting average patient characteristics has been utilized. ${ }^{43}$ Such analysis is possible when individual patient data are known in one trial and can be adjusted for comparison with a second trial that does not describe individual patient data. In comparing a trial of ADA versus PBO with one of etanercept versus PBO, adjustments were made for the ADA trial's lower mean age, greater prevalence of PsA, less prior use of systemic treatment or phototherapy, and smaller percentage of body surface area affected. The results showed that, compared with the etanercept-treated patients, an additional 17.2\% of the ADA-treated patients experienced a PASI score $\geq 75 \%$ ( $P<0.001)$, and that the mean percentage PASI score improvements from baseline were greater for ADAtreated than etanercept-treated patients at weeks 4,8 , and 12 (all $P<0.05$ ).

\section{Crohn's disease and JIA}

Head-to-head trials directly comparing the efficacy of two biologics in these two conditions have not been published. Indirect comparisons have also not been performed.

\section{Adverse events}

A Cochrane review found that adverse events associated with the nine biologic drugs used in rheumatology, compared with controls, had a significantly higher rate of total adverse events (also specifically the case for ADA and infliximab), withdrawals because of adverse events (specifically for infliximab), and tuberculosis reactivation. ${ }^{44}$ This analysis was of 163 controlled trials, over 50,000 participants, and had a short median duration of 6 months. When considering all serious adverse events as a group, ADA had no increase in risk compared with PBO, the four other anti-TNF drugs (etanercept, infliximab, certolizumab, and golimumab), abatacept, rituximab, anakinra, and tocilizumab. This Cochrane review was limited by the fact that $63 \%$ of papers did not clearly define what constitutes a serious adverse event. The most significant events considered here will be serious infections, lymphoma and other malignancies, injection site reactions, congestive heart failure (CHF), demyelination, induction of autoimmunity, and serious hepatic events.

Serious infections are a potential concern with all potent immunomodulators, and include tuberculosis, bacterial infections such as pneumonia, osteomyelitis, sepsis, soft tissue infections, and viral infections (most seriously the reactivation of hepatitis $\mathrm{B}$ virus). The most detailed report to date was a 2010 systematic review of serious infections in 209 trials and extension studies from biologic medications. ${ }^{44}$ Considered as a group, biologic medications did not result in increased odds of a serious infection (in most cases defined as deaths, hospitalizations, and use of intravenous antibiotics), (odds ratio [OR], 1.19; 95\% CI, 0.94-1.26). Abatacept was the only biologic with a point estimate trending toward lower infection risk than control (OR, 0.57; 95\% CI, 0.30-1.08), ADA was among the seven biologics showing a nonsignificant trend toward higher infection risk (OR, 1.12; 95\% CI, 0.73-1.70), while certolizumab was the only agent with a statistically significant increase in risk (OR, 3.51; 95\% CI, 1.59-7.79). Direct evidence of an increased risk of serious infection also comes from a British prospective cohort registry of over 15,000 RA patients. ${ }^{45}$ Here, the anti-TNF drugs ADA, etanercept, and infliximab were associated with a significantly increased risk of infection compared with oral DMARDs (adjusted HR, 1.2; 95\% CI, 1.1-1.5), with no statistically significant difference in risk between the three anti-TNF drugs.

The labels of ADA and the other anti-TNF medications specifically mention risk of tuberculosis. The Cochrane review found that the risk of reactivation of tuberculosis is elevated when studies of nine biologics are combined (OR, 4.68; 95\% CI, 1.18-18.60), but the review did not have enough data to comment on individual drug risk. ${ }^{44}$ A large observational study using a British registry of over 
10,000 RA patients who were receiving ADA, etanercept, or infliximab was compared with 3232 RA controls receiving oral DMARDs. ${ }^{46}$ Here, ADA showed a statistically significant increased risk of tuberculosis compared with etanercept (adjusted incidence rate ratio, 4.1; 95\% CI, 1.4-12.4), with a similar trend for infliximab compared with etanercept (incidence rate ratio, $3.1 ; 95 \% \mathrm{CI}, 1.0-9.5)$. The median time to diagnosis of tuberculosis was 13.4 months.

An elevated risk of lymphoma has been recognized in RA patients since before the era of biologic medications. ${ }^{47}$ In one of the largest systematic reviews, Singh et $\mathrm{al}^{44}$ found a pooled risk of lymphoproliferative malignancies for nine biologics used in rheumatology to have a statistically nonsignificant OR of 0.53 (95\% CI, 0.17-4.28). Askling et $\mathrm{al}^{48}$ reported an individual patient meta-analysis based on 74 trials of etanercept, infliximab, and ADA, and found that $0.84 \%$ of patients on anti-TNF drugs and $0.64 \%$ of controls developed a malignancy during the clinical trials (RR, 0.99; 95\% CI, 0.61-1.68, nonsignificant). Other large Swedish and German cohorts have also found no increase in solid cancer or hematologic malignancies, as well as no increase in cancer incidence with increasing duration of use of anti-TNF antibody drugs. ${ }^{49-51}$ On the other side of the ledger, two studies reported an increased rate of lymphoma. A Swedish study of 1557 patients found a HR of 4.9 (95\% CI, 0.9-2.62) for those on anti-TNF medications compared with those not taking anti-TNF drugs; however, this did not reach statistical significance. ${ }^{52}$ An Italian study of 1064 patients reported an increased lymphoma risk in those taking anti-TNF medication, but this report did not employ as high a quality of outcome ascertainment as found in other studies. ${ }^{53}$ For ADA specifically, studies using pooled data in 10,041 RA patients and 3160 Crohn's disease patients found no statistically significant increase in malignancies when compared with control populations. ${ }^{54,55}$ However, in the case of nonmelanoma skin cancer, a meta-analysis of 74 studies using anti-TNF drugs for at least 4 weeks has found a significantly increased RR (RR, 2.02; 95\% CI, 1.11-3.95). ${ }^{48}$ In 2009 the FDA issued a warning about the risk of cancers in children and adolescents receiving anti-TNF medications for JIA, Crohn's disease, or other inflammatory disorders. ${ }^{56}$ The warning was based on 48 reports of malignancies, about half of which were lymphomas, some of which were rare and aggressive hepatosplenic T-cell lymphomas. Nevertheless, there are insufficient pediatric data that control for autoimmune disease characteristics, concomitant medication use (such as azathioprine), and cancer incidence rates in control populations.
In clinical practice, rheumatologists discuss the risks of malignancy with patients but they can also point out that more than 10 years' clinical experience has not demonstrated a definite increased rate of hematopoietic or solid tumors except for nonmelanoma skin cancers. For many patients, the possibility of improved disease control and quality of life is a powerful counterweight to the small or absent increase in risk of malignancy.

New or worsening CHF is listed as a possible adverse event in the product insert for anti-TNF products. A retrospective analysis of Medicare data did indeed find an increased risk for hospitalization because of $\mathrm{CHF}$ in RA patients receiving anti-TNF drugs rather than MTX (HR, 1.70; 95\% CI, 1.07-2.69). ${ }^{57}$ However, several studies do not confirm this observation. A recent systematic review and meta-analysis of cohort studies found that anti-TNF $\alpha$ therapy was associated with a reduced risk for all cardiovascular events including CHF (pooled adjusted RR, 0.46; 95\% CI, 0.28-0.77). ${ }^{58}$ A large British registry assessed the risk of myocardial infarction in responders to anti-TNF treatment compared with nonresponders after 6 months of therapy. Those responding to anti-TNF drugs had a reduced rate of 3.5 events per 1000 patient years compared with 9.4 events per 1000 patient years. ${ }^{59}$ The cardiovascular risks of RA and its treatment is a field of intense study and additional data are emerging annually.

Information on other serious adverse events associated with ADA is based on cases observed during regulatory trials and case reports or series. Induction of autoimmune features such as antinuclear antibodies can be quite common. For example, in Crohn's disease treated with infliximab, the rate of antinuclear antibody positivity was $56.8 \%$ after 24 months. ${ }^{60}$ Anti-double-stranded DNA antibodies and antihistone antibodies are less common but are also observed, while a clinical lupus-like syndrome is unusual. ${ }^{61}$ New-onset psoriasis, especially palmoplantar pustulosis, has been described in RA patients treated with anti-TNF drugs. ${ }^{62}$ Demyelination, such as Guillain-Barré syndrome or worsening of multiple sclerosis, has been described since the early days of anti-TNF drug use, and serious hepatic events, including elevation of liver function tests, are described but are not proven as cause and effect. ${ }^{63,64}$ Injection site reactions occur in an estimated $17.5 \%$ of ADA-treated patients and $22.4 \%$ of etanercepttreated patients. ${ }^{65}$ These reactions generally consist of pain, skin rash, pruritus, and erythema and are therefore not severe. By contrast, $0.5 \%$ of infliximab infusions result in severe infusion reactions, and such reactions contribute to 
a higher rate of drug discontinuation for infliximab than either ADA or etanercept. ${ }^{66,67}$

\section{Advantages and disadvantages of using ADA \\ Advantages}

In considering the advantages of using ADA, the effectiveness of the product is at the top of the list (Table 3). There is no disagreement about ADA's overall effectiveness in achieving clinical responses and radiographic stabilization (where relevant). Second, physicians have at least 10 years of experience with the product and patients are reassured that ADA's potential side effects are well recognized in most cases. Third, there is the additional advantage that blood test monitoring is less extensive than it would be for other DMARDs such as MTX. Fourth, ADA has a relatively long duration of action and therefore has a convenient every-2-week dosing interval in most applications. Fifth, ADA can be considered in patients with liver (eg, hepatitis C), renal, or lung comorbidities, in situations where the toxicities of MTX or leflunomide would be unacceptable.

\section{Disadvantages}

There are also disadvantages to ADA that need to be factored into considerations for its clinical use. The first factor to consider is the high cost of not only ADA but also all the other biologics medications, with ADA now retailing at US\$1950 for 1 month of therapy using $40 \mathrm{mg}$ eow. ${ }^{68}$ This dwarfs the costs of all other clinical expenses for RA patients, except when hospitalization is needed. Second, although ADA has impressive clinical efficacy in $60 \%-70 \%$ of cases in multiple conditions, the flip side is that $30 \%-40 \%$ of patients do not respond adequately and need a different therapy. Furthermore, loss of effectiveness well into a course of therapy may in part be due to the formation of anti-ADA antibodies in some patients.

Table 3 Advantages and disadvantages of adalimumab (ADA) use

Advantages
I. Effectiveness
2. Convenient dosing interval
3. I0-year track record
4. Straightforward monitoring for side effects
5. Availability for hepatitis $C$ patients
Disadvantages
1. Cost
2. Ineffectiveness in subset
3. Requires refrigeration and use of needles
4. Contraindicated with recurrent infections and congestive heart failure
5. Anti-ADA antibodies may form

Third, some patients are reluctant to change from oral DMARDs to injected ADA, perceiving it as a dramatic escalation of their therapy. Patients accept injected medications for cancer or for diabetes but do not commonly realize that injections have become standard therapy for multiple autoimmune conditions. Fourth, injected ADA has the disadvantage of requiring refrigeration during shipment and storage, and medications with needles require extra planning for those passing through airport security checks. Finally, ADA use is not appropriate for all candidates, as multiple comorbidities limit use in patients with, for example, chronic or recurrent infections, CHF, or demyelination. In this regard, a hopeful point appears to be that as further studies are performed and longer post-marketing surveillance occurs, the use of ADA may increase in certain cases found to have acceptable risks upon closer study (eg, hepatitis C, hepatitis B with viral prophylaxis, and CHF).

\section{Conclusion}

In the end, ADA is part of a class of five available anti-TNF $\alpha$ medications with broadly similar therapeutic effectiveness and side effect profiles. A lack of head-to-head clinical trials comparing the anti-TNF drugs means that no definite advantage has been convincingly demonstrated for any single product. An increasing number of comparative effectiveness reports and meta-analyses are being published in an effort to infer small differences in clinical effectiveness. Nevertheless, the main high-quality data for such studies still stem from the pivotal randomized, controlled trials that originally led to drug approval.

In choosing a TNF $\alpha$ inhibitor, the clinician moves beyond considerations of effectiveness and major side effects, which do not differentiate the products sufficiently. Instead, the decision to use ADA may be driven more by the convenience of twice-a-month dosing, the familiarity of the physician with the product's 10-year track record, or the ready availability of a manufacturer's program to defray the co-pay or the entire drug costs. In clinical practice, it is becoming less common to treat refractory disease activity sequentially with more than two different TNF $\alpha$ inhibitors, indicating that there is a stable or growing market for biologics targeting other aspects of inflammatory pathways ( $\mathrm{T}$ - and B-cell viability or activation, IL-1, IL-6, IL-17, and others). In addition, the reluctance of many patients to use injected medications means that future products such as orally administered small molecule inhibitors (eg, Janus kinase inhibitors) could significantly cut into the market share of biologic drugs. Biosimilars, which are biologics manufactured from the same DNA sequence 
as the original product but by a different company, have been extremely slow to advance, as no guidelines have been developed for proving equivalent efficacy and safety.

ADA is expected to have a leading role in the treatment of RA and other inflammatory conditions for years to come. When there are beneficial results in about $65 \%$ of patients (eg, the ACR20 response in the ARMADA trial of ADA in $\mathrm{RA}$ ), there is a need for alternate therapies in the $35 \%$ of patients who do not respond initially or the further patients who later lose their response. Future studies will need to address the optimal sequence of DMARDs and biologics to use, combinations of DMARDs and biologics, and headto-head comparisons of biologics in clinical trials. For those who go into clinical remission on an anti-TNF medication, unanswered questions remain about identifying the patients who can maintain the remission off all drugs, or at least off injected medication. Given the cost of biologic drugs, even studies that increase the interval between drug doses in wellcontrolled patients could provide financial benefits.

\section{Disclosure}

This material is the result of work supported with resources and the use of facilities at the Dallas VA Medical Center. The views expressed in this article are those of the author and do not necessarily reflect the position or policy of the Department of Veterans Affairs or the United States government. Dr Reimold has been an investigator for clinical trials sponsored by Argos Therapeutics, Eli Lilly and Company, Genentech, Human Genome Sciences, Janssen Biotech, Medimmune, and Novartis.

\section{References}

1. European Medicines Agency. Humira: EPAR - product information. 2009 [updated May 19, 2011]. Available from: http://www.ema.europa. eu/ema/index.jsp?curl=pages/includes/document/document_detail.jsp? webContentId=WC500050870\&mid=WC0b01ac058009a3dc. Accessed December 20, 2011.

2. Bartelds GM, Krieckaert CLM, Nurmohamed MT, et al. Development of antidrug antibodies against adalimumab and association with disease activity and treatment failure during long-term follow-up. JAMA. 2011;305(14):1460-1468.

3. Weinblatt ME, Keystone EC, Furst DE, et al. Adalimumab, a fully human anti-tumor necrosis factor monoclonal antibody, for the treatment of rheumatoid arthritis in patients taking concomitant methotrexate: the ARMADA trial. Arthritis Rheum. 2003;48(1):35-45.

4. Van de Putte LB, Atkins C, Malaise M, et al. Efficacy and safety of adalimumab as monotherapy in patients with rheumatoid arthritis for whom previous disease modifying antirheumatic drug treatment has failed. Ann Rheum Dis. 2004;63(5):508-516.

5. Keystone EC, Kavanaugh AF, Sharp JT, et al. Radiographic, clinical, and functional outcomes of treatment with adalimumab (a human anti-tumor necrosis factor monoclonal antibody) in patients with active rheumatoid arthritis receiving concomitant methotrexate therapy: a randomized, placebo-controlled, 52-week trial. Arthritis Rheum. 2004;50(5):1400-1411.
6. Furst DE, Schiff MH, Fleischmann RM, et al. Adalimumab, a fully human anti tumor necrosis factor-alpha monoclonal antibody, and concomitant standard antirheumatic therapy for the treatment of rheumatoid arthritis: results of STAR (Safety Trial of Adalimumab in Rheumatoid Arthritis). J Rheumatol. 2003;30(12):2563-2571.

7. Breedveld FC, Weisman MH, Kavanaugh AF, et al; for the PREMIER Investigators. The PREMIER study: a multicenter, randomized, double-blind clinical trial of combination therapy with adalimumab plus methotrexate versus methotrexate alone or adalimumab alone in patients with early, aggressive rheumatoid arthritis who had not had previous methotrexate treatment. Arthritis Rheum. 2006;54(1):26-37.

8. Mease PJ, Gladman DD, Ritchlin CT, et al; for the Adalimumab Effectiveness in Psoriatic Arthritis Trial Study Group. Adalimumab for the treatment of patients with moderately to severely active psoriatic arthritis: results of a double-blind, randomized, placebo-controlled trial. Arthritis Rheum. 2005;52(10):3279-3289.

9. Genovese MC, Mease PJ, Thomson GT, et al; for the M02-570 Study Group. Safety and efficacy of adalimumab in treatment of patients with psoriatic arthritis who had failed disease modifying antirheumatic drug therapy. J Rheumatol. 2007;34(5):1040-1050.

10. Van der Heijde, Kivitz A, Schiff MH, et al; for the ATLAS Study Group. Efficacy and safety of adalimumab in patients with ankylosing spondylitis: results of a multicenter, randomized, double-blind, placebocontrolled trial. Arthritis Rheum. 2006;54(7):2136-2146.

11. Hanauer SB, Sandborn WJ, Rutgeerts P, et al. Human anti-tumor necrosis factor monoclonal antibody (adalimumab) in Crohn's disease: the CLASSIC-1 trial. Gastroenterology. 2006;130(2):323-333.

12. Sandborn WJ, Rutgeerts P, Enns R, et al. Adalimumab induction therapy for Crohn disease previously treated with infliximab: a randomized trial. Ann Intern Med. 2007;146(12):829-838.

13. Colombel JF, Sandborn WJ, Rutgeerts P, et al. Adalimumab for maintenance of clinical response and remission in patients with Crohn's disease: the CHARM trial. Gastroenterology. 2007;132(1):52-65.

14. Menter A, Tyring SK, Gordon K, et al. Adalimumab therapy for moderate to severe psoriasis: a randomized, controlled phase III trial. J Am Acad Dermatol. 2008;58(1):106-115.

15. Saurat JH, Stingl G, Dubertret L, et al; for the CHAMPION Study Investigators. Efficacy and safety results from the randomized controlled comparative study of adalimumab vs methotrexate vs placebo in patients with psoriasis (CHAMPION). Br J Dermatol. 2008;158(3):558-566.

16. Lovell DJ, Ruperto N, Goodman S, et al; for the Pediatric Rheumatology Collaborative Study Group and Pediatric Rheumatology International Trials Organisation. Adalimumab with or without methotrexate in juvenile rheumatoid arthritis. N Engl J Med. 2008;359(8):810-820.

17. Weinblatt ME, Keystone EC, Furst DE, Kavanaugh AF, Chartash EK, Segurado OG. Long term efficacy and safety of adalimumab plus methotrexate in patients with rheumatoid arthritis: ARMADA 4 year extended study. Ann Rheum Dis. 2006;65(6):753-759.

18. Keystone E, van der Heijde D, Weinblatt ME, et al. Initial combination therapy with adalimumab plus methotrexate leads to better long-term outcomes in patients with advanced rheumatoid arthritis: analysis of the final 10-year results of an open-label extension of a phase 3 trial. Arthritis Rheum. 2011;63(Suppl 10):S870-S871.

19. Breedveld FC, Keystone E, van der Heijde D, et al. Initial combination therapy with adalimumab plus methotrexate leads to better longterm outcomes than with either monotherapy in patients with early rheumatoid arthritis: 8-year results of an open-label extension of a phase 3 trial. Arthritis Rheum. 2011;63(Suppl 10):S481.

20. Mease PJ, Ory P, Sharp JT, et al. Adalimumab for long-term treatment of psoriatic arthritis: 2-year data from the Adalimumab Effectiveness in Psoriatic Arthritis Trial (ADEPT). Ann Rheum Dis. 2009;68(5):702-709.

21. Saad AA, Ashcroft DM, Watson KD, et al; for the British Society for Rheumatology Biologics Register. Persistence with anti-tumour necrosis factor therapies in patients with psoriatic arthritis: observational study from the British Society of Rheumatology Biologics Register. Arthritis Res Ther. 2009;11(2):R52. 
22. Sieper J, van der Heijde D, Dougados M, Brown LS, Lavie F, Pangan AL. Early response to adalimumab predicts long-term remission through 5 years of treatment in patients with ankylosing spondylitis. Ann Rheum Dis. Epub November 29, 2011.

23. Van der Heijde D, Salonen D, Weissman BN, et al; for the Canadian (M03-606) study group and ATLAS study group. Assessment of radiographic progression in the spines of patients with ankylosing spondylitis treated with adalimumab for up to 2 years. Arthritis Res Ther. 2009;11(4):R127.

24. Van der Heijde D, Pangan AL, Schiff MH, et al; for the ATLAS study group. Adalimumab effectively reduces the signs and symptoms of active ankylosing spondylitis in patients with total spinal ankylosis. Ann Rheum Dis. 2008;67(9):1218-1221.

25. Kamm MA, Hanauer B, Panaccione R, et al. Adalimumab sustains steroid-free remission after 3 years of therapy for Crohn's disease. Aliment Pharmacol Ther. 2011;34(3):306-317.

26. Chaparro M, Panés J, Garcia V, et al. Long-term durability of response to adalimumab in Crohn's disease. Inflamm Bowel Dis. Epub May 25, 2011.

27. Schiff M, Keiserman M, Codding C, et al. Efficacy and safety of abatacept or infliximab vs placebo in ATTEST: a phase III, multicentre, randomised, double-blind, placebo-controlled study in patients with rheumatoid arthritis and an inadequate response to methotrexate. Ann Rheum Dis. 2008;67(8):1096-1103.

28. Markenson JA, Gibofsky A, Palmer WR, et al. Persistence with anti-tumor necrosis factor therapies in patients with rheumatoid arthritis: observations from the RADIUS registry. $J$ Rheumatol. 2011;38(7):1273-1281

29. Kievit W, Adang EM, Fransen J, et al. The effectiveness and medication costs of three anti-tumour necrosis factor alpha agents in the treatment of rheumatoid arthritis from prospective clinical practice data. Ann Rheum Dis. 2008;67(9):1229-1234.

30. Kuriya B, Arkema EV, Bykerk VP, Keystone EC. Efficacy of initial methotrexate monotherapy versus combination therapy with a biological agent in early rheumatoid arthritis: a meta-analysis of clinical and radiographic remission. Ann Rheum Dis. 2010;69(7): $1298-1304$.

31. Turkstra E, Ng SK, Scuffham PA. A mixed treatment comparison of the short-term efficacy of biologic disease modifying anti-rheumatic drugs in established rheumatoid arthritis. Curr Med Res Opin. 2011;27(10):1885-1897.

32. Nixon R, Bansback N, Brennan A. The efficacy of inhibiting tumour necrosis factor alpha and interleukin 1 in patients with rheumatoid arthritis: a meta-analysis and adjusted indirect comparisons. Rheumatology (Oxford). 2007;46(7):1140-1147.

33. Wiens A, Venson R, Correr CJ, Otuki MF, Pontarolo R. Meta-analysis of the efficacy and safety of adalimumab, etanercept, and infliximab for the treatment of rheumatoid arthritis. Pharmacotherapy. 2010;30(4):339-353.

34. Kievit W, Fransen J, Oerlemans AJ, et al. The efficacy of antiTNF in rheumatoid arthritis, a comparison between randomised controlled trials and clinical practice. Ann Rheum Dis. 2007;66(11): 1473-1478.

35. Saad AA, Symmons DP, Noyce PR, Ashcroft DM. Risks and benefits of tumor necrosis factor-alpha inhibitors in the management of psoriatic arthritis: systematic review and metaanalysis of randomized controlled trials. J Rheumatol. 2008;35(5):883-890.

36. Migliore A, Bizzi E, Broccoli S, Laganà B. Indirect comparison of etanercept, infliximab, and adalimumab for psoriatic arthritis: mixed treatment comparison using placebo as common comparator. Clin Rheumatol. 2012;31(1):133-137.

37. Kirstensen LE, Karlsson JA, Englund M, Petersson IF, Saxne T, Geborek P. Presence of peripheral arthritis and male sex predicting continuation of anti-tumor necrosis factor therapy in ankylosing spondylitis: an observational prospective cohort study from the South Swedish Arthritis Treatment Group Register. Arthritis Care Res (Hoboken). 2010;62(10):1362-1369.
38. Glintborg B, Ostergaard M, Krogh NS, Dreyer L, Kristensen HL, Hetland ML. Predictors of treatment response and drug continuation in 842 patients with ankylosing spondylitis treated with antitumour necrosis factor: results from 8 years' surveillance in the Danish nationwide DANBIO registry. Ann Rheum Dis. 2010;69(11): 2002-2008

39. Arends S, Lebbink HR, Spoorenberg A, et al. The formation of autoantibodies and antibodies to TNF- $\alpha$ blocking agents in relation to clinical response in patients with ankylosing spondylitis. Clin Exp Rheumatol. 2010;28(5):661-668.

40. Spadaro A, Punzi L, Marchesoni A, et al. Switching from infliximab or etanercept to adalimumab in resistant or intolerant patients with spondyloarthritis: a 4-year study. Rheumatology (Oxford). 2010;49(6):1107-1111.

41. Zhang Z, Schmitt J, Wozel G, Kirch W. Treatment of plaque psoriasis with biologics: a meta-analysis of randomized controlled trials. Med Klin (Munich). 2009;104(2):125-136. German.

42. Reich K, Burden AD, Eaton JN, Hawkins NS. Efficacy of biologics in the treatment of moderate to severe psoriasis: a network meta-analysis of randomized controlled trials. Br J Dermatol. 2012;166(1):179-188.

43. Signorovitch JE, Wu EQ, Yu AP, et al. Comparative effectiveness without head-to-head trials: a method for matching-adjusted indirect comparisons applied to psoriasis treatment with adalimumab or etanercept. Pharmacoeconomics. 2010;28(10):935-945.

44. Singh JA, Wells GA, Christensen R, et al. Adverse effects of biologics: a network meta-analysis and Cochrane overview. Cochrane Database Syst Rev. 2011;(2):CD008794.

45. Galloway JB, Hyrich KL, Mercer LK, et al; for the BSRBR Control Centre Consortium and British Society for Rheumatology Biologics Register. Anti-TNF therapy is associated with an increased risk of serious infections in patients with rheumatoid arthritis especially in the first 6 months of treatment: updated results from the British Society for Rheumatology Biologics Register with special emphasis on risks in the elderly. Rheumatology (Oxford). 2011;50(1):124-131.

46. Dixon WG, Hyrich KL, Watson KD, et al. Drug-specific risk of tuberculosis in patients with rheumatoid arthritis treated with anti-TNF therapy: results from the British Society for Rheumatology Biologics Register (BSRBR). Ann Rheum Dis. 2010;69(3):522-528.

47. Baecklund E, Ekbom A, Sparén P, Feltelius N, Klareskog L. Disease activity and risk of lymphoma in patients with rheumatoid arthritis: nested case-control study. BMJ. 1998;317(7152):180-181.

48. Askling J, Fahrbach K, Nordstrom B, Ross S, Schmid CH, Symmons D. Cancer risk with tumor necrosis factor alpha (TNF) inhibitors: meta-analysis of randomized controlled trials of adalimumab, etanercept, and infliximab using patient level data. Pharmacoepidemiol Drug Saf. 2011;20(2):119-130.

49. Strangfeld A, Hierse F, Rau R, et al. Risk of incident or recurrent malignancies among patients with rheumatoid arthritis exposed to biologic therapy in the German biologics register RABBIT. Arthritis Res Ther. 2010;12(1):R5.

50. Askling J, Fored CM, Brandt L, et al. Risks of solid cancers in patients with rheumatoid arthritis and after treatment with tumour necrosis factor antagonists. Ann Rheum Dis. 2005;64(10):1421-1426.

51. Askling J, Fored CM, Baecklund E, et al. Haematopoietic malignancies in rheumatoid arthritis: lymphoma risk and characteristics after exposure to tumour necrosis factor antagonists. Ann Rheum Dis. 2005;64(10):1414-1420.

52. Geborek P, Bladström A, Turesson C, et al. Tumour necrosis factor blockers do not increase overall tumour risk in patients with rheumatoid arthritis, but may be associated with an increased risk of lymphomas. Ann Rheum Dis. 2005;64(5):699-703.

53. Pallavicini FB, Caporali R, Sarzi-Puttini P, et al. Tumour necrosis factor antagonist therapy and cancer development: analysis of the LORHEN registry. Autoimmun Rev. 2010;9(3):175-180

54. Burmester GR, Mease P, Dijkmans BA, et al. Adalimumab safety and mortality rates from global clinical trials of six immune-mediated inflammatory diseases. Ann Rheum Dis. 2009;68(12):1863-1869. 
55. Colombel JF, Sandborn WJ, Panaccione R, et al. Adalimumab safety in global clinical trials of patients with Crohn's disease. Inflamm Bowel Dis. 2009;15(9):1308-1319.

56. US Food and Drug Administration. FDA: cancer warnings required for TNF blockers [press release]. Silver Spring, MD: US Food and Drug Administration; [August 4, 2009]. Available from: http://www.fda. gov/NewsEvents/Newsroom/PressAnnouncements/ucm175803.htm. Accessed April 10, 2012.

57. Setoguchi S, Schneeweiss S, Avorn J, et al. Tumor necrosis factor-alpha antagonist use and heart failure in elderly patients with rheumatoid arthritis. Am Heart J. 2008;156(2):336-341.

58. Barnabe C, Martin BJ, Ghali WA. Systematic review and meta-analysis: anti-tumor necrosis factor $\alpha$ therapy and cardiovascular events in rheumatoid arthritis. Arthritis Care Res (Hoboken). 2011;63(4):522-529.

59. Dixon WG, Watson KD, Lunt M, et al. Reduction in the incidence of myocardial infarction in patients with rheumatoid arthritis who respond to anti-tumor necrosis factor alpha therapy: results from the British Society for Rheumatology Biologics Register. Arthritis Rheum. 2007;56(9):2905-2912.

60. Vermeire S, Noman M, Van Assche G, et al. Autoimmunity associated with anti-tumor necrosis factor alpha treatment in Crohn's disease: a prospective cohort study. Gastroenterology. 2003;125(1):32-39.

61. Schiff MH, Burmester GR, Kent JD, et al. Safety analyses of adalimumab (Humira) in global clinical trials and US postmarketing surveillance of patients with rheumatoid arthritis. Ann Rheum Dis. 2006;65(7):889-894.
62. Harrison MJ, Dixon WG, Watson KD, et al; for the British Society for Rheumatology Biologics Register Control Centre Consortium. Rates of new-onset psoriasis in patients with rheumatoid arthritis receiving anti-tumour necrosis factor alpha therapy: results from the British Society for Rheumatology Biologics Register. Ann Rheum Dis. 2009;68(2):209-215.

63. Mohan N, Edwards ET, Cupps TR, et al. Demyelination occurring during anti-tumor necrosis factor alpha therapy for inflammatory arthritides. Arthritis Rheum. 2001;44(12):2862-2869.

64. Suissa S, Ernst P, Hudson M, BittonA, KezouhA. Newer disease-modifying antirheumatic drugs and the risk of serious hepatic adverse events in patients with rheumatoid arthritis. Am J Med. 2004;117(2):87-92.

65. Gartlehner G, Thieda P, Morgan LC, Thaler K, Hansen RA, Jonas B. Drug Class Review: Targeted Immune Modulators; Final Report Update 2. PMID: 21089249. Portland: Oregon Health and Science University; Nov 2009. Available from: http:/www.ncbi.nlm.nih.gov/pubmedhealth/ PMH0008734/pdf/TOC.pdf. Accessed April 10, 2012.

66. Du Pan SM, Dehler S, Ciurea A, Ziswiler HR, Gabay C, Finckh A; for the Swiss Clinical Quality Management Physicians. Comparison of drug retention rates and causes of drug discontinuation between anti-tumor necrosis factor agents in rheumatoid arthritis. Arthritis Rheum. 2009;61(5):560-568.

67. Schaible TF. Long term safety of infliximab. Can J Gastroenterol. 2000;14 Suppl C:29C-32C.

68. Drugstore.com [homepage on the Internet]. Available from: http://www drugstore.com/. Accessed January 10, 2012.
Open Access Rheumatology Research and Reviews

\section{Publish your work in this journal}

Open Access Rheumatology Research and Reviews is an international, peer-reviewed, open access journal, publishing all aspects of clinical and experimental rheumatology in the clinic and laboratory including the following topics: Pathology, pathophysiology of rheumatological diseases; Investigation, treatment and management of rheumatological

\section{Dovepress}

diseases; Clinical trials and novel pharmacological approaches for the treatment of rheumatological disorders. The manuscript management system is completely online and includes a very quick and fair peerreview system, which is all easy to use. Visit http://www.dovepress.com/ testimonials.php to read real quotes from published authors. 